Ann. Génét. Sél. anim., I970, 2 (2), Ir9-I27.

\title{
EXPRESSIVITÉ DES FACTEURS DE PANACHURE LIMITÉE CHEZ LE CHEVAL DOMESTIQUE
}

\author{
Ph. DREUX \\ Laboratoire de Zoologie, \\ École normale supérieure, 24, rue Lhomond, Paris-Ve
}

\section{RÉSUMÉ}

Dans la race des chevaux trotteurs français, une statistique quantitative de l'étendue des marques blanches permet de préciser l'expressivité des gènes mis en jeu, en fonction de la couleur de la robe. Cette expressivité, toujours plus forte chez les alezans, est beaucoup plus variable pour les marques faciales que pour les balzanes.

\section{INTRODUCTION}

Nous avons fait connaître dans un précédent article (DREUX, I966 a) les résultats d'une statistique des phénotypes sur 4095 poulains représentant en I 957 , I 958 et I959 la production dans la race du cheval trotteur français. C'était là un travail préliminaire pour une étude qui a pour but d'essayer d'élucider la génétique des marques blanches limitées (marques en tête, balzanes) chez le Cheval domestique. En même temps avaient été recueillies 384 fratries de 4 poulains au moins qui forment un excellent matériel pour cette étude, dont il est facile de prévoir la complexité.

En étudiant ces fratries, il est rapidement apparu que l'expressivité variable des facteurs en jeu demandait un travail préliminaire pour arriver à répondre à la plus simple des questions : à savoir le classement d'un individu comme possédant une marque ou non. En effet, qu'il s'agisse de marques faciales ou de balzanes, on observe une variation continue depuis la présence d'une forte tache blanche jusqu'à sa réduction à une très petite zọne dépigmentée et sa disparition totale. La statistique déjà citée faisait prévoir dẹ plus que l'expression d'un même génotype de panachure dépendait dans une certaine mesure de la couleur de la robe de 1'individu. 
Il a donc été nécessaire de procéder à une étude statistique quantitative des différentes marques blanches en tenant compte de la couleur du fond de la robe. Ces préoccupations avaient déjà amené WALTHER (I9I3) à un important travail qui avait montré que la robe alezan amène une expression plus forte des marques blanches. Ses conclusions ne répondent pas au but que nous nous sommes fixé car elles concernaient d'autres races, de patrimoine génétique moyen probablement différent; c'est également le cas du travail de GEURTs (I969) sur la race du cheval frison.

En raison du matériel utilisé, les croisements expérimentaux sont impossibles et l'on est forcé de se contenter de ceux qui sont effectués par les éleveurs. Ce problème est donc de résolution très difficile et toutes les méthodes qui peuvent 1'éclaircir sont les bienvenues. On a été conduit à utiliser entre autres celles de la génétique des populations et à les appliquer à plusieurs reprises à la race des trotteurs français qui constitue un ensemble à peu près fermé de 20000 individus environ. L'étude des fratries recueillies a montré que cet ensemble peut être considéré comme une population panmictique par rapport aux caractères qui nous intéressent, en d'autres termes que les éleveurs ne font pas plus volontiers de croisements entre des chevaux très marqués par exemple, ou peu marqués, ou entre des chevaux de robe déterminée (DREux, r969), et ceci apparemment depuis l'origine de la race du trotteur français.

Le fait que l'étendue moyenne des marques diffère suivant la couleur du fond de la robe peut être interprété de deux façons différentes :

- association entre les gènes amenant les marques et les gènes gouvernant la couleur du fond de la robe;

- les gènes gouvernant la couleur du fond de la robe sont des modificateurs de l'expressivité des gènes amenant les marques.

La première hypothèse est à écarter pour les raisons suivantes :

- la panmixie est constatée pour chacun des deux groupes de caractères; les éleveurs n'attachent aucune importance à ces critères pour effectuer les croisements, et encore moins à la combinaison des deux facteurs;

- les fratries étudiées s'étendent sur une quarantaine d'années et cette panmixie existe donc depuis un nombre de générations suffisant pour qu'on puisse considérer la population comme en équilibre;

- l'effectif de la population est suffisant (DREUX, I966 a) pour que la consanguinité ne soit pas trop marquée;

- enfin, la liaison statistique des marques les plus étendues en moyenne avec les robes les plus claires est un fait qui a été constaté dans toutes les races de chevaux examinées jusqu'ici avec quelque détail (entre autres WALTHER, I9I3; GEURTS, I969) quoique en général sans données quantitatives précises.

Il semble donc vraisemblable d'admettre dans ce qui suit que les gènes de panachure et ceux du fond de la robe ont dans cette population une répartition statistique indépendante. Par exemple, l'ensemble des chevaux alezans présentera la même fréquence des différents gènes inconnus gouvernant la panachure limitée que l'ensemble des chevaux bais, ou des chevaux bai-brun. 


\section{COTATION QUANTITATIVE DES MARQUES}

Une fois obtenus ces résultats préliminaires, on pourra comparer l'expressivité de l'ensemble des facteurs de panachure en fonction de la couleur de la robe. Pour cela, on fera dans chacune des trois catégories de robe; alezan, bai, bai-brun ou plus foncé (DREUX, Ig66 a) une statistique quantitative de l'étendue des marques blanches. En principe, la robe alezane correspond à l'homozygote $b b$ (CASTLE, r954), bien que cette homologie puisse être discutée. La robe baie correspond au phénotype $[A B]$. Les chevaux bai-brun et plus foncés, pouvant aller jusqu'au noir, correspondent aux phénotypes $\left[a^{\mathrm{t}} a^{\mathrm{t}} B\right]\left[a^{\mathrm{t}} a B\right],[a a B]$ ou peut-être dans certains cas à la présence de 1'allèle $E^{D}$ (CASTlE et Singleton, I960; DreuX, I966 b).

Dans des études de ce genre, la méthode la plus rationnelle est d'évaluer pour chaque individu le pourcentage de la surface panachée. Un bon exemple de travail de ce genre est celui de DUNN et LITrLE, sur la Souris, résumé par GRÜNEBERG (I943). La méthode n'est malheureusement pas applicable au Cheval car elle exigerait l'examen personnel de milliers d'individus dispersés à travers la France, et il faut bien se contenter de travailler seulement sur leurs signalements détaillés. On a donc été amené à définir une cotation arbitraire des marques qui sont heureusement décrites de façon précise dans le signalement.

Il est d'ailleurs bon de remarquer au passage que la méthode consistant à définir le pourcentage de la surface panachée n'est pas elle-même à l'abri de toute critique, car les différents territoires du corps ont certainement à ce point de vue une valeur différente; et on ne peut évaluer celle-ci qu'après une étude très approfondie.

Les marques blanches se situent à la face où elles se développent généralement à partir d'une tache frontale, jusqu'à l'envahir complètement; et aux membres en commençant par leurs extrémités. Pour les marques faciales, BLuNN et HoWELL (I936) avaient défini 4 territoires qu'ils jugeaient (à tort d'ailleurs) indépendants, mais qui correspondent à des régions assez naturelles et que nous avons adoptées dans notre statistique préliminaire (DREUX, I966 a). Ce sont : l'étoile au front, la liste sur le chanfrein, le ladre aux naseaux et à la lèvre supérieure, le ladre à la lèvre inférieure. Nous avons attribué à chacun de ces territoires une cotation de o à 2; o désignant l'absence totale de marque, $x$ une trace très petite, 2 une marque bien développée. Pour l'ensemble de la face, la cotation s'étend. donc de o (absence de toute marque en tête) jusqu'à 8 (marques bien développées dans les 4 terrritoires).

Pour les marques aux membres, le signalement détaillé d'un cheval emploie une série de termes traditionnels définissant de façon assez précise l'étendue de la balzane; on y a fait correspondre arbitrairement la cotation suivante :

0 : absence totale de balzane.

I : traces (petites taches isolées contiguës au sabot).

2 : principe, entourant la couronne sans la dépasser.

3 : petite balzane, remontant sur le paturon.

4 : balzane, couvrant le boulet.

5 : grande balzane, s'étendant sur le canon. 
6 : balzane chaussée, atteignant le carpe ou le tarse.

7 : balzane haut chaussée, empiétant sur l'avant-bras ou la jambe.

8 : balzane très haut chaussée, arrivant près du corps.

Dans notre statistique, la cote 6 est rare, les cotes 7 et 8 exceptionnelles. Les cotes sont additionnées pour les quatre membres et peuvent donc varier pour un individu de $o$ (aucune marque) jusqu'à 32 (balzanes très haut chaussées aux quatre membres); pratiquement elles n'ont pas dépassé 24 .

L'évaluation de la panachure totale s'est faite en additionnant les cotes des balzanes et des marques faciales, en l'absence de toute donnée sur leur importance respective permettant par exemple d'affecter les secondes d'un coefficient par rapport aux premières.

L'étude statistique de la cotation des marques suivant la couleur de la robe n'a tenu compte que du phénotype de celle-ci; en effet, la distinction entre bais homozygotes et hétérozygotes pour le gène $B$, possible dans certains cas, ne l'est pas toujours. Elle ne semble pas d'ailleurs avoir d'utilité, au moins en première approximation (WALTHER, I9I3).

Ces cotations quantitatives ont été appliquées à l'étude du même matériel que dans notre statistique préliminaire, c'est-à-dire l'ensemble des poulains trotteurs nés en 1957 , I958 et I959, donnant un échantillonnage objectif de cette population. Les deux sexes ont été groupés pour ne pas trop compliquer le travail; ils présentent cependant des différences marquées mais petites; 1'interprétation ne peut encore en être envisagée (DREUX, r966 a). L'échantillonnage comprenait 4095 poulains : I 338 alezans, 2002 bais, 755 bai-brun ou plus foncés; les quelques rares sujets grisonnés ont été réunis à la catégorie de leur robe de base.

\section{MARQUES TOTALES}

Pour chacune des trois robes de base, on a construit un diagramme donnant la répartition de la panachure en portant en abscisses sa cotation définie plus haut et en ordonnées les pourcentages de chaque classe. Les tableaux donnant les nombres exacts des effectifs correspondants auraient exigé trop de place pour être présentés, et la représentation graphique a paru suffisante.

Le diagramme de panachure totale des alezans (I 338 individus) (fig. I) apparaît très différent de celui des bais ( 2002 individus) (fig. $\mathrm{I} b$ ) et de celui des bai-brun (755 individus) (fig. I c); ces deux derniers au contraire sont similaires;

Les maxima intermédiaires des distributions correspondent à des phénotypes très fréquents: mis en évidence dans notre statistique précédente (DREUX, I966 a): Par exemple celui de. la cotation 2 représente dans sa très grande majorité les chevaux dont la seule marque est une étoile frontale; 6 correspond à une étoile et une balzane d'extension moyenne 4 très fréquente; Io à une étoile et deux balzanes, en général postérieures; I4 et I6, maxima importants chez les alęzans, à une marque faciale de forte extension 6 et à deux balzanes moyennes ou grandes.

On ne s'étendra pas trop sur ces maxima intermédiaires dont l'intérêt est diminué par le caractère arbitraire des cotations; on en retiendra seulement le 
fait que certaines combinaisons de marques sont plus communes que d'autres. La principale utilité de ces diagrammes est leur contribution pour distinguer dans les fratries les phénotypes dépourvus de marques (que les hippologues désignent souvent sous le terme traditionnel de zain) de ceux qui en présentent.
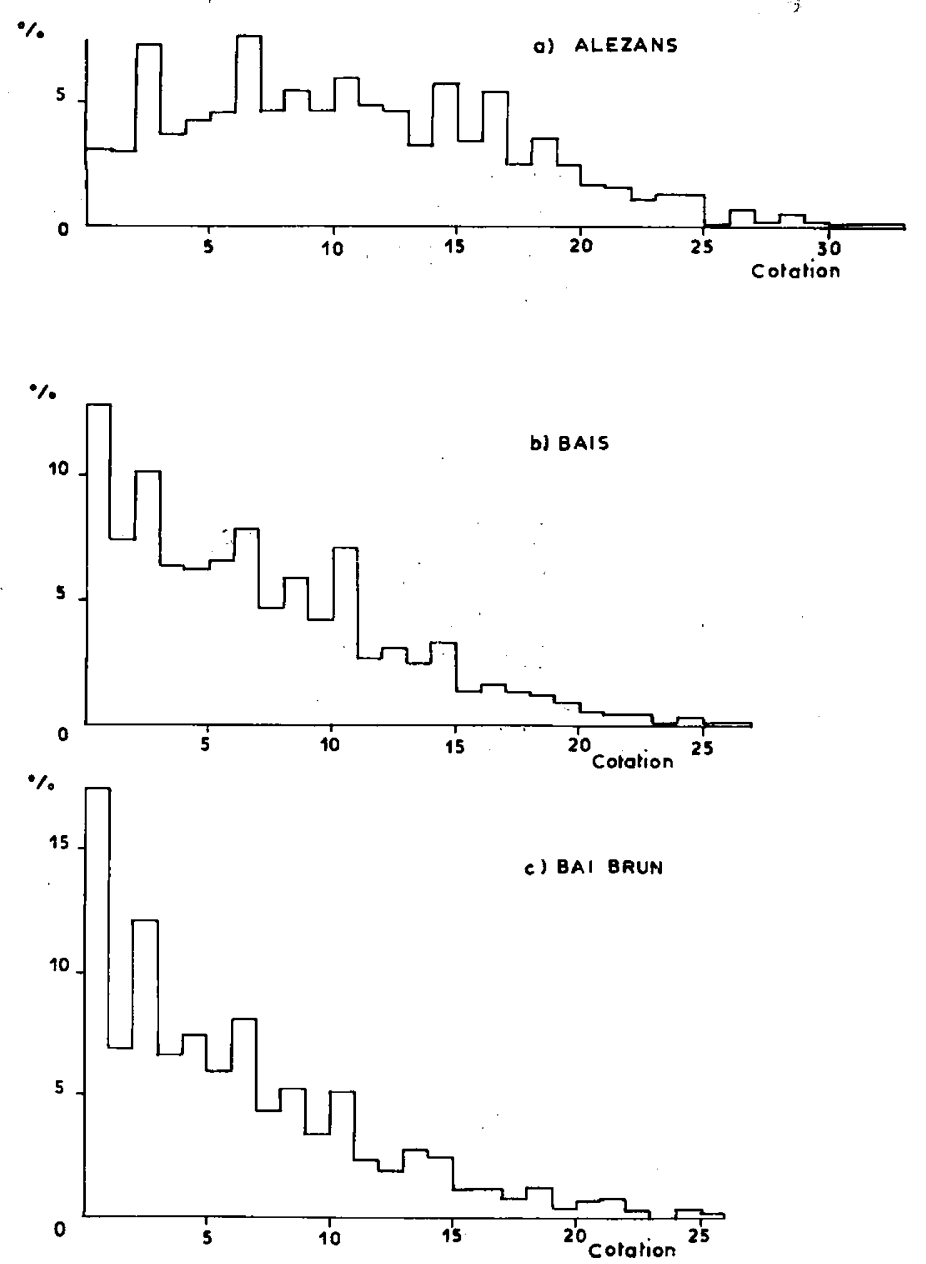

Frg. r. - Distribution de l'ensemble des marques blanches chez le trotteur français (en abscisses la cotation des marques, en ordonnées le pourcentage de chaque classe).
a) $\mathrm{r}_{33} 8$ chevaux de phénotype alezan.
b) 2002 chevaux de phénotype bai.
c) 755 chevaux de phénotype bai-brun.

Cette distinction est facile pour les bais et bai-brun où le maximum de cote $o$ (respectivement $\mathrm{I} 2,8 \%$ et $\mathrm{I} 7,4 \%$ ) est largement séparé du maximum de cote 2 correspondant pour sa majorité à une étoile frontale. Le minimum de cote I correspond à une très petite touffe frontale de poils blancs ou moins fréquemment à une trace de balzane à un pied généralement postérieur et ne peut être classé de façon absolue. Si l'on tient compte du fait admis plus haut que la dis- 
tribution des génotypes de panachure se fait au hasard indépendamment de la robe, on conçoit qu'il faudra être plus sévère pour classer un bai-brun comme non marqué qu'un bai. La situation est différente pour les alezans où les individus sans marques sont bien moins nombreux $(3, \mathrm{I} \%)$ et il est facile de concevoir qu'un génotype qui n'amènerait aucune marque chez un bai donnera au contraire chez un alezan une marque de cotation moyenne 2, c'est-à-dire dans la majorité des cas une étoile frontale; ce maximum 2 est nettement séparé du suivant de cote 6 qui correspond grossièrement au maximum de cote 2 chez les bais et bai-brun.

L'expression globale des facteurs de panachure est donc bien supérieure chez les alezans. On peut admettre avec vraisemblance qu'elle est due en fait à la pénétrance plus forte de certains des gènes mis en jeu chez l'homozygote $b b$. L'étude séparée des deux sortes de marques apportera quelques précisions.

\section{MARQUES EN TÊTE}

La figure $2(a, b, c)$ représente les distributions des marques en tête en fonction de leur cotation chez les alezans, les bais et les bai-brun. Les diagrammes des bais et bai-brun sont là aussi très semblables entre eux, mais diffèrent beaucoup de celui des alezans. Les cotes de rang impair sont beaucoup moins nombreuses que celles de rang pair car les marques en tête sont décrites sur les signalements des chevaux avec moins de précision que les balzanes et ce n'est que rare-

a) ALEZANS
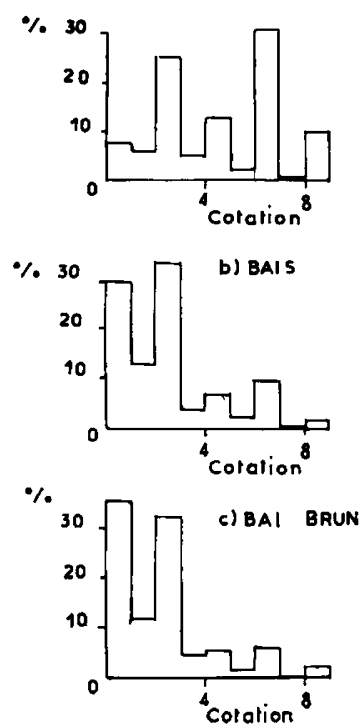

Fig. 2. - Distribution des marques en tête chez le trotteur français.

a) 1338 chevaux de phénotype alezan.

b) 2002 chevaux de phénotype bai.

c) 755 chevaux de phénotype bai-brun. 
ment que l'on peut coter avec sécurité une marque comme très petite; sauf heureusement pour la cote I qui représente dans plus de $97 \%$ des cas (Dreux, I966 a) une petite touffe frontale de poils blancs.

Ces distributions précisent les constatations précédemment faites sur l'ensemble des marques. Les chevaux sans marques en tête se présentent en proportion voisine chez les bais $(39,5 \%)$ et les bai-brun $(35,2 \%)$. Au contraire très peu d'alezans $(7,8 \%)$ en sont tout à fait dépourvus; un génotype amenant en général une étoile frontale chez un alezan donnera en général une absence totale de marque en tête chez un cheval bai ou plus foncé. Inversement, un génotype amenant chez un alezan une marque faciale très développée, de cote 6 à 8 , n'amènera dans la majorité des cas qu'une simple étoile frontale chez un bai ou un bai-brun.

Les différences d'expressivité liées à la robe sont ici très importantes et on voit qu'en étudiant les fratries on devra en tenir le plus grand compte. On admettra par exemple une équivalence approchée au point de vue du génotype de panachure entre un bai sans marque et un alezan à étoile frontale, entre un alezan avec une marque de cote 6 (notée ELNo dans notre précédente statistique, DREUX I966 a) et un bai avec une étoile frontale.

\section{BALZANES}

Les figures $3 a$, $b$ et $c$ montrent les distributions des balzanes en fonction de leur cotation respectivement chez les alezans, les bais et les bai-brun. On remarquera que cette fois les trois distributions se ressemblent beaucoup.

Un premier maximum, le plus élevé, correspond à l'absence totale de balzanes; il est de 20, I p. Ioo chez les alezans, 28,0 p. Ioo chez les bais, 32,9 p. Ioo chez les bai-brun. Là encore l'expressivité des facteurs de panachure aux membres est variée, mais beaucoup moins que pour les marques en tête; on pourrait interpréter ce résultat en admettant qu'un nombre moindre de facteurs entre en jeu que pour ces dernières, mais d'autres causes peuvent $y$ contribuer.

Le second maximum correspond clairement à une seule balzane (très généralement postérieure), et le maximum le plus éle vé ensuite à deux balzanes, le plus souvent postérieures.

On peut séparer ici de façon très nette les individus sans balzanes ou avec une seule trace, de cote $I$, de ce ux qui possèdent au moins une balzane bien développée. Malheureusement il est fac ile de se rendre compte qu'une certaine proportion des génotypes amenant l'absence de balzane sur un cheval bai ou plus foncé en produira une au moins sur un alezan; et ceci peut amener de grandes difficultés dans les études de fratries, si elles ne sont pas de robe homogène.

\section{CONCLUSION}

L'objet pratique de ce travail était d'apporter des éléments objectifs a fin de pouvoir séparer de la façon la plus nette possible chez le cheval, les phénotypes portant des marques (faciales ou aux membres) de ceux qui n'en portent pas, 
de manière à faciliter l'étude des fratries recueillies. Èn effet, la variation d'étendue de ces marques est assez continue et seule une discontinuité dans la distribution permet d'y faire des coupures.
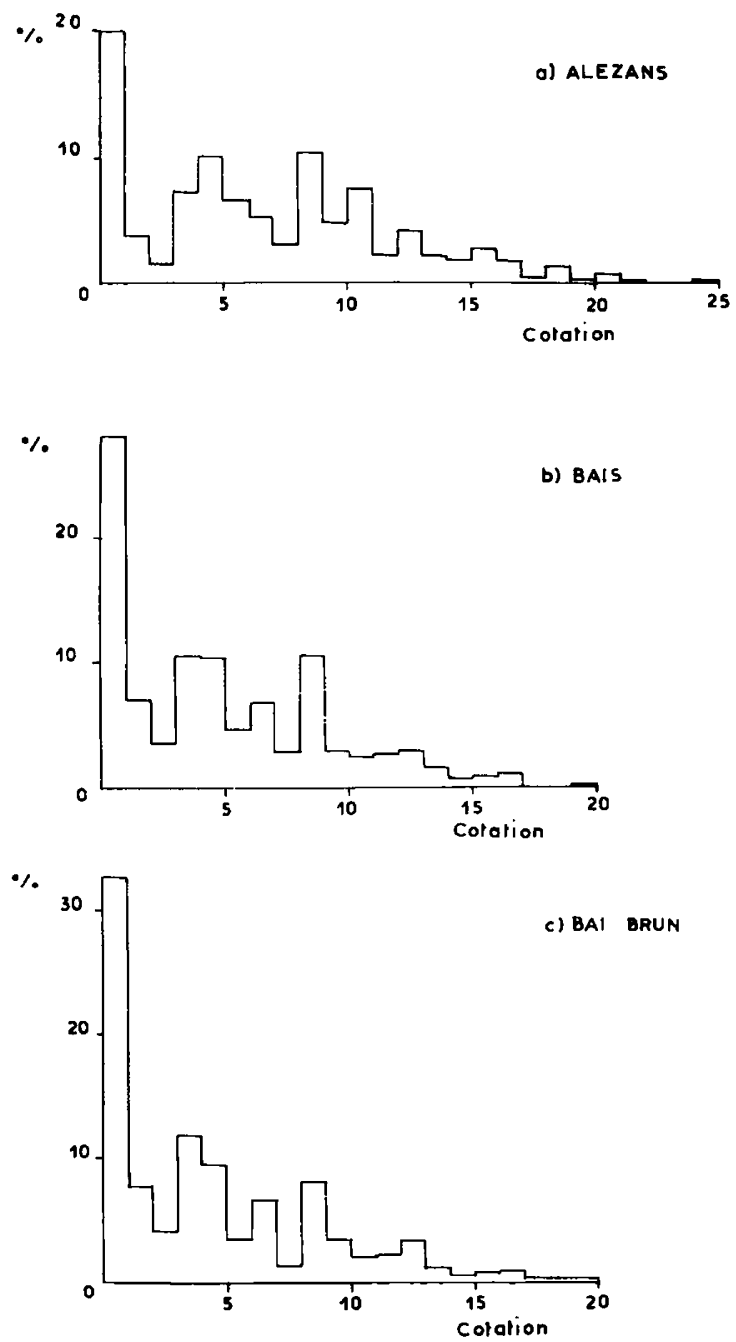

Fig. 3. - Distribution des balzanes chez le trotteur français

a) 1338 chevaux de phénotype alezan.

b) 2002 chevaux de phénotype bai.

c) 755 chevaux de phénotype bai-brun.

On a vu dans les pages précédentes que les distributions des marques ne sont pas trop différentes chez les bais et les chevaux plus foncés. Au contraire, les alezans (homozygotes $b b$ ), présentent une distribution similaire pour les balzanes (avec toutefois une proportion plus faible de phénotypes non marqués) mais tout à fait différente pour les marques faciales et qui se répercute naturellement sur la distribution de l'ensemble des marques. 
Ceci rend d'autant plus difficile l'étude génétique de fratries hétérogènes pour la robe (au moins quant au gène $B$ ); celles-ci sont malheureusement les plus nombreuses. On est donc amené à rechercher une homologie moyenne entre les marques chez les alezans et chez les bais et bai-brun. La comparaison des distributions permet de la faire d'une façon approchée mais qui n'a rien d'absolu; et il est encore très difficile de préciser les variations d'expressivité d'un génotype donné.

La race des trotteurs français étant assimilable à une population panmictique pour les facteurs étudiés ici, il est légitime d'admettre que les gènes des marques et les gènes de couleur se comportent indépendamment dans la population. Les distributions différentes des cotations quantitatives sont donc dues à l'influence des seconds sur l'expressivité et la pénétrance des premiers.

Reçu pour publication en janvier r97o.

\section{SUMMARY}

\section{EXPRESSIVITY OF FACTORS FOR IIMITED PIEBALDNESS IN THE DOMESTIC HORSE}

The French trotter breed may be considered as a random mating population for the characters of coat color and variegation (DREUX, 1969). The distribution of the two groups of genes controlling these characters in the population are independent.

In a sampling of 4095 individuals, facial marking ranges from zero (absence) to 8 (maximum), and each white stocking from zero (absence) to 8 (maximum). The distribution in the sampling of these markings was traced for each type of color : chestnut, bay, brown bay, and darker (CAstle and Singleton, 1960), or : bb, AB, and $a^{t} a^{t} B-a^{t} a b-a a B-E^{D}$.

Figure $1(a, b, c)$ shows these distributions for all the markings. Figure $2(a, b, c)$ shows only facial markings. Figure $3(\mathrm{a}, \mathrm{b}, \mathrm{c})$ shows only white stocking markings. By comparing them, the differences in expression of variegation factors due to genes controlling coat color may be determined.

\section{RÉFÉRENCES BIBLIOGRAPHIQUES}

BLUNN C.T., Howell C.E., 1936. -The inheritance of white facial markings in Arabian horses. J. Hered., 27, 293-299.

CASTLE W.E., I954. Coat color inheritance in horses and in other mammals. Genetics, 39, 35-44.

CAstle W.E., Singleton W.R., I960. Genetics of the "brown" horse. J. Heved., 51, 127-I 30.

Dreux Ph., r966 a. Introduction statistique à la génétique des marques blanches limitées chez le cheval domestique. Ann. Génét., 9, 66-72.

Dreux Ph., I966 b. Contribution à l'étude du gène $E$ chez le cheval domestique. Ann. Génét., 9, I68-r7o.

DREUx Ph., 1969. La panmixie dans une race de chevaux domestiques : l'exemple des trotteurs français. Ann. Génét. Sél. anim., 1, 184-185.

GEURTS R.H.J.J., I969. Genetische analyse en structur van de fokkerij van het Friese paard. I4I p. Thèse Méd. vet. Utrecht.

GRÜNEBERG H., 1943. The genetics of the mouse. $4 \mathrm{r} 2 \mathrm{p}$. Cambridge University Press.

WALther A., I9I3. Die Vererbung unpigmentierter Haare und Hautstellen bei Rind und Pferd. Ztschr. indukt. A bst. Vererbsl. 10, $\mathrm{r}-48$. 